\title{
The ectodomain of Toll-like receptor 9 is cleaved to generate a functional receptor
}

\author{
Sarah E. Ewald ${ }^{1}$, Bettina L. Lee ${ }^{1}$, Laura Lau ${ }^{1}$, Katherine E. Wickliffe ${ }^{1}$, Guo-Ping Shi ${ }^{2}$, Harold A. Chapman ${ }^{3}$ \\ $\&$ Gregory M. Barton ${ }^{1}$
}

\begin{abstract}
Mammalian Toll-like receptors (TLRs) 3, 7, 8 and 9 initiate immune responses to infection by recognizing microbial nucleic acids $^{1,2}$; however, these responses come at the cost of potential autoimmunity owing to inappropriate recognition of self nucleic acids ${ }^{3}$. The localization of TLR9 and TLR7 to intracellular compartments seems to have a role in facilitating responses to viral nucleic acids while maintaining tolerance to self nucleic acids, yet the cell biology regulating the transport and localization of these receptors remains poorly understood ${ }^{4-6}$. Here we define the route by which TLR9 and TLR7 exit the endoplasmic reticulum and travel to endolysosomes in mouse macrophages and dendritic cells. The ectodomains of TLR9 and TLR7 are cleaved in the endolysosome, such that no full-length protein is detectable in the compartment where ligand is recognized. Notably, although both the full-length and cleaved forms of TLR9 are capable of binding ligand, only the processed form recruits MyD88 on activation, indicating that this truncated receptor, rather than the full-length form, is functional. Furthermore, conditions that prevent receptor proteolysis, including forced TLR9 surface localization, render the receptor non-functional. We propose that ectodomain cleavage represents a strategy to restrict receptor activation to endolysosomal compartments and prevent TLRs from responding to self nucleic acids.
\end{abstract}

The subcellular localization of TLR9 and TLR7 is highly regulated. Although ligand recognition occurs in endolysosomes, it has been reported that most, if not all, TLR9 resides in the endoplasmic reticulum (ER) of resting cells ${ }^{5,6}$. Curiously, analyses of N-linked glycosylation on TLR9 indicates that the protein does not travel through the Golgi, even in activated cells ${ }^{5,6}$. These observations have led to a model in which TLR9 is recruited directly to endolysosomes from the ER, bypassing the classical secretory pathway ${ }^{5}$. However, general mechanistic details accounting for such unusual cell biology are lacking, and the specific details of how TLR9 transport is regulated between these compartments remain largely unknown.

To separate ER-resident TLR9 from receptors in the endolysosome, we purified phagosomes from cells fed latex beads. To track TLR9, we used macrophage-like RAW264 cells stably expressing TLR9 with a carboxy-terminal haemagglutinin (HA) tag (RAWTLR9 cells). We have previously shown that this epitope tag does not affect TLR9 function ${ }^{4}$. As expected, phagosome preparations were enriched for the lysosomal protein LAMP1 whereas they were devoid of markers of other intracellular organelles and the cytosol (Fig. 1a). Surprisingly, we did not observe any TLR9 at the expected molecular mass $(150 \mathrm{kDa})$. Instead, an approximately $80 \mathrm{kDa}$ protein was highly enriched in the phagosomal preparation (Fig. 1a). The presence of ligand did not alter receptor translocation, as we could not detect full-length TLR9 in phagosomes containing latex beads coated with CpG oligonucleotides (Fig. 1b), despite the fact that these beads induced TLR9 signalling (Supplementary Fig. 1a). In contrast, the $80 \mathrm{kDa}$ protein was evident in phagosomes at the earliest time point examined $(10 \mathrm{~min})$ and remained equally abundant over $4 \mathrm{~h}$ (Fig. 1b).

To demonstrate directly that the $80 \mathrm{kDa}$ band in our phagosome preparations represented a cleaved form of TLR9, we performed pulse-chase analysis. The $80 \mathrm{kDa}$ band was first detectable after $3 \mathrm{~h}$ of chase, concomitant with the onset of full-length receptor degradation (Fig. 1c). The $80 \mathrm{kDa}$ band continued to accumulate in parallel with the degradation of full-length TLR9, peaking at $6 \mathrm{~h}$ when the fulllength protein was no longer detectable. These data formally demonstrate that the $80 \mathrm{kDa}$ protein is a cleavage product of full-length TLR9 consisting of approximately half of the ectodomain, the transmembrane domain and the entire cytoplasmic domain. On the basis of molecular mass, we estimate that cleavage occurs within or distal to leucine-rich repeat (LRR) 14. Structural modelling of TLR9 suggests that the cleaved receptor adopts an abbreviated 'horseshoe' fold encompassing a region that corresponds to residues implicated in ligand binding by TLR3 (refs 7, 8) (Fig. 1d). A C-terminal cleavage product of TLR9 was also present in macrophage colony-stimulating factor (M-CSF)-derived macrophages, granulocyte-macrophage (GM)-CSF-derived dendritic cells, and Flt3L-derived plasmacytoid dendritic cells, indicating that similar processing events occur in many immunologically relevant cell types (Fig. 1e). We observed a similar cleavage product of TLR7, but not of TLR4, indicating that receptor processing may be a general feature shared by nucleic-acid-sensing TLRs (Fig. 1f).

We next sought to determine where within the cell TLR9 cleavage occurs. Although $\mathrm{N}$-linked sugars on full-length TLR9 were sensitive to endoglycosidase $\mathrm{H}$ (EndoH), most of the sugars on the cleaved form of TLR9 were resistant to EndoH, indicating that the truncated form of the receptor has passed through the Golgi (Fig. 2a). A similar glycosylation pattern was observed for TLR7 (Supplementary Fig. 1b). In addition, a small fraction of full-length TLR9 consistently migrated slightly slower than most of the full-length protein (Fig. 2b, see asterisk). Notably, this high-molecular-mass band was almost entirely EndoH resistant, in contrast to the rest of the full-length TLR9 protein, indicating that this small pool of full-length protein has also passed through the Golgi. We also observed this high-molecular-mass, EndoH-resistant band in pulse-chase experiments (asterisks in Fig. 1c and Supplementary Fig. 1c). The band appeared after $1.5 \mathrm{~h}$ of chase, immediately preceding formation of the cleavage product. Furthermore, the intensity of the high-molecular-mass band appeared to match the intensity of the accumulated cleavage product.

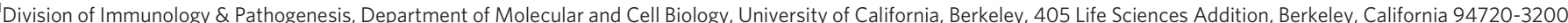

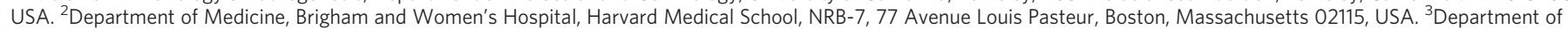
Medicine, and The Cardiovascular Research Institute, University of California, San Francisco, Box 0111, San Francisco, California 94143, USA. 
a

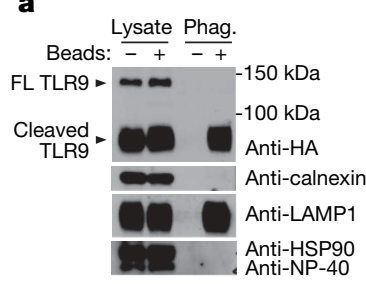

b Lysate

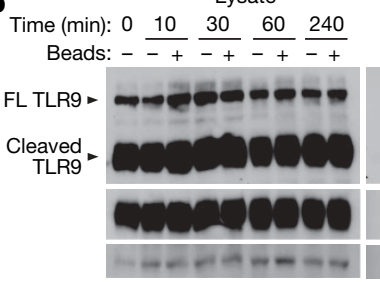

Phagosome

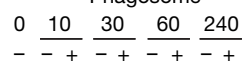

- $-\frac{30}{-+} \frac{60}{-+} \frac{240}{-+}$

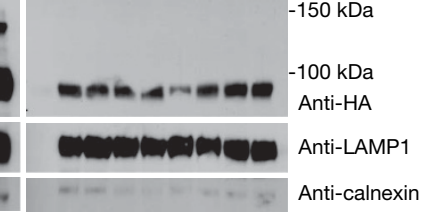

LRR15

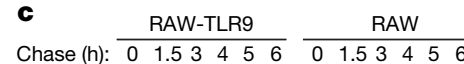

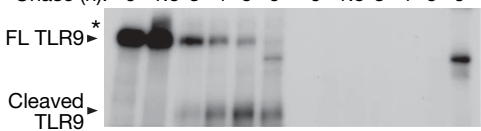

$150 \mathrm{kDa}$

NS $-100 \mathrm{kDa}$ (

\section{Figure $1 \mid$ A truncated version of TLR9 is exclusively present in}

phagosomes. a, Lysates or phagosome preparations from RAW-TLR9 cells fed latex beads were separated by SDS-PAGE and probed with antibodies specific for the indicated proteins. Full-length TLR9 (FL TLR9) and the truncated protein (cleaved TLR9) are indicated. b, Analysis of phagosomes from RAW-TLR9 cells fed unconjugated latex beads or beads conjugated to stimulatory $\mathrm{CpG}$ oligonucleotides. Lysates or purified phagosomes from the cells were separated by SDS-PAGE and probed with antibodies against the indicated proteins. c, The truncated form of TLR9 is a product of the fulllength protein. Pulse-chase analysis of RAW-TLR9 cells or control cells (RAW). An asterisk indicates the high-molecular-mass full-length TLR9 band. NS indicates a nonspecific band present in both RAW-TLR9 and

Collectively, these data suggest that this high-molecular-mass band represents the small fraction of TLR9 that exits the ER, passes through the Golgi, and is cleaved. In support of this model, cells treated with brefeldin A (BFA), a reagent that disrupts the Golgi, contained neither the high-molecular-mass band (1.5-h chase) nor the $80-\mathrm{kDa}$ cleavage product (5-h chase) (Fig. 2c). Analysis of TLR9 localization by immunofluorescence microscopy corroborated our biochemical findings (Supplementary Figs 2 and 3).

To address the mechanism responsible for TLR9 sorting from the ER, we examined UNC93B1 (refs 9-11). Mice with a deficiency in UNC93B1 no longer respond to TLR3, TLR7 and TLR9 ligands ${ }^{11}$, and UNC93B1 can associate with TLR3, TLR7 and TLR9 which may facilitate transport to endolysosomes ${ }^{9,10}$. We generated RAW cells in which Unc93b1 mRNA was knocked down by retrovirally encoded short hairpin RNA (shRNA) (Fig. 3a). As expected, these cells exhibited reduced TNF- $\alpha$ production in response to $\mathrm{CpG}$ and $\mathrm{R} 848$ relative to control cells (Fig. 3b). Notably, the TLR9 cleavage product was greatly reduced in UNC93B1 knockdown cells, although full-length TLR9 was expressed at levels comparable to control cells (Fig. 3c). The high molecular mass, full-length TLR9 band was also absent in UNC93B1 knockdown cells (Fig. 3c), and TLR9 was not present in phagosomes purified from UNC93B1 knockdown cells (Fig. 3d). These data demonstrate that UNC93B1 is required for TLR9 exit from the ER. To test whether UNC93B1 is also sufficient for this exit, we overexpressed UNC93B1 in mouse embryonic fibroblasts (MEFs), which signal poorly in response to $\mathrm{CpG}$ oligonucleotides. Whereas we detected very little TLR9 cleavage in control cells, simply overexpressing UNC93B1 in MEFs resulted in a 75-fold increase in the $80-\mathrm{kDa}$ band (Fig. 3e). The increase in cleaved TLR9 corresponded with a robust increase in responsiveness to $\mathrm{CpG}$ oligonucleotides (Fig. 3f). Thus, UNC93B1 seems to control the functional pool of TLR9 by regulating exit from the ER. Furthermore, the fraction of TLR9 that exits the ER and is cleaved seems to be responsible for TLR9 signalling.

We next attempted to block TLR9 cleavage by inhibiting various cellular proteases. Bafilomycin A1, an inhibitor of the vacuolar ATPase, blocks endolysosomal acidification as well as signalling by TLR9, TLR7 and TLR3 (ref. 12). Pulse-chase analysis of RAW-TLR9 cells treated with bafilomycin A1 revealed that TLR9 cleavage was control RAW cells. d, Modelled structure of the truncated ectodomain (residues 477-790) of TLR9. Leucine-rich repeats (LRR) 15-26 are labelled. e, The TLR9 cleavage product is present in multiple cell types.

Haematopoietic stem cells were transduced with a retrovirus encoding TLR9-HA and then differentiated into macrophages with M-CSF (MØ), dendritic cells with GM-CSF (cDC), or plasmacytoid dendritic cells with Flt3L (pDC). f, Lysates from RAW cells expressing TLR4-HA or TLR7-HA were probed with anti-HA antibody. Note that TLR4 is not cleaved. The upper band of TLR4 is due to glycosylation. Unless indicated otherwise, the data presented in each panel are representative of at least three independent experiments.

completely blocked when endolysosomal acidification was inhibited (Fig. 4a). Blocking TLR9 proteolysis with baflomycin A1 also resulted
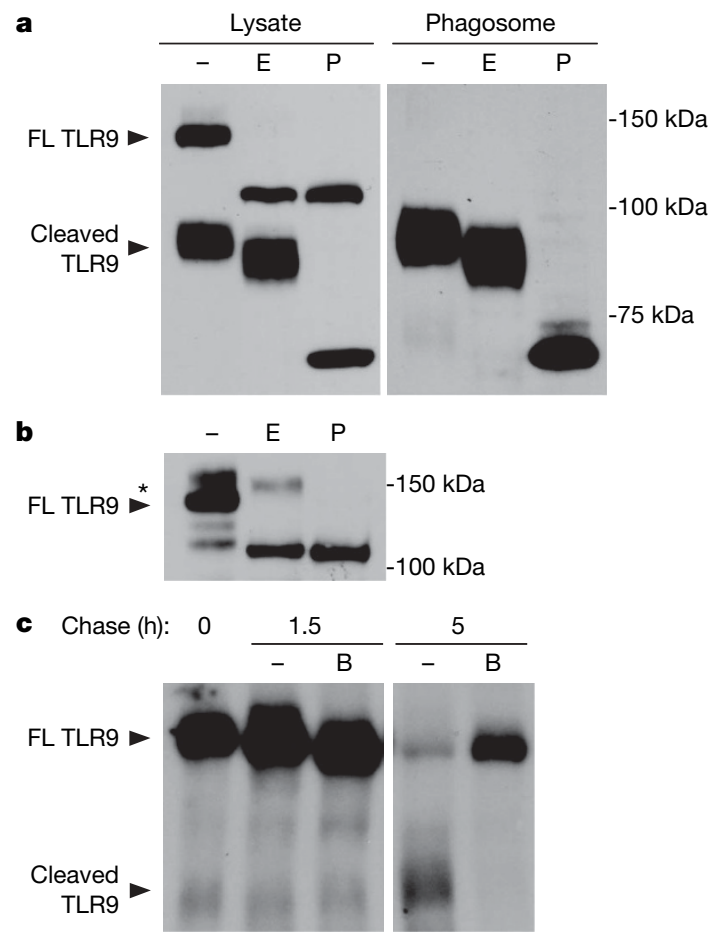

Figure 2 | TLR9 exits the ER and travels through the Golgi before cleavage. a, Cleaved TLR9 is EndoH-resistant. Proteins from lysates or purified phagosomes from RAW-TLR9 cells were immunoprecipitated with anti-HA resin and treated with EndoH (E), PNGaseF (P), or left untreated (-). b, A longer exposure of TLR9 immunoprecipitated from RAW-TLR9 cells and subjected to analysis as described in a. An asterisk indicates the highmolecular-mass full-length TLR9 band that is EndoH-resistant. c, TLR9 cleavage requires passage through the Golgi. Pulse-chase analysis was performed, at the indicated chase time points, on RAW-TLR9 cells treated with brefeldin A (B) or vehicle (-). The data presented in each panel are representative of at least three experiments. 


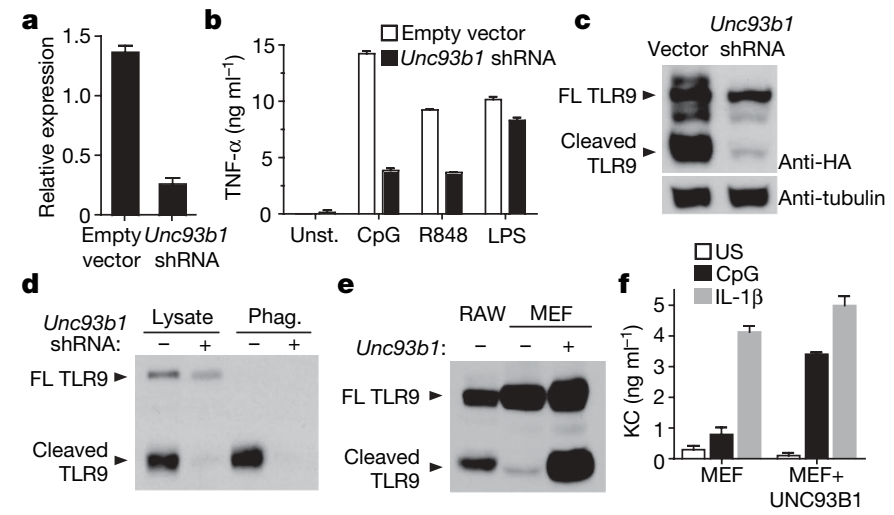

Figure 3 | UNC93B1 is required for TLR9 ER exit and cleavage. a, Expression of $U n c 93 b 1$ transcript was measured by quantitative RT-PCR in RAW cells transduced with a control retrovirus or a retrovirus encoding an shRNA specific for Unc93b1. b, Unc93b1 shRNA RAW cells respond poorly to TLR7 and TLR9 ligands but not TLR4 ligands. RAW cells or Unc93b1 shRNA RAW cells were stimulated with the indicated ligands and TNF- $\alpha$ production was measured by ELISA (representative of two experiments). LPS, lipopolysaccharide; Unst., unstimulated. c, UNC93B1 is required for TLR9 cleavage. Lysates from control RAW-TLR9 cells (vector) or RAW-TLR9 cells transduced with the Unc $93 b 1$ shRNA retrovirus were probed with anti-HA antibodies or anti-tubulin antibodies. d, Lysates or purified phagosomes from the same cells described in c were analysed for the presence of TLR9 by anti-HA immunoblot. e, Overexpression of UNC93B1 in MEFs enhances TLR9 cleavage. TLR9 in lysates of MEFs stably expressing TLR9-HA or TLR9-HA with UNC93B1 was detected by anti-HA immunoblot. RAW-TLR9 cells were included as a control for TLR9 cleavage product. f, Cells described in e were stimulated with $5 \mu \mathrm{M} \mathrm{CpG}$ or $25 \mathrm{ng} \mathrm{ml}^{-1}$ recombinant mouse IL-1 $\beta$ or left unstimulated (US). CXCL1/KC (KC) production was measured by ELISA (representative of two experiments). Unless noted otherwise, all data presented are representative of at least three experiments. Error bars represent standard error.

in a 20-fold accumulation of the high-molecular-mass TLR9 species, further supporting the idea that this band is the precursor to the cleavage product.

Two recent reports have shown that inhibitors of cathepsin $\mathrm{K}$ (CTSK) and cathepsin B (CTSB) block TLR9 signal transduction in dendritic cells and macrophages, respectively ${ }^{13,14}$. To address whether specific cathepsins may be involved in TLR9 processing, we tested whether recombinant cathepsins could cleave TLR9 in vitro. We observed that recombinant CTSK and CTSS could cleave full-length TLR9 in vitro to generate an approximately $80-\mathrm{kDa}$ cleavage product (Fig. 4b). Although these experiments implicate cathepsin family members in TLR9 processing, we were unable to block processing using cathepsin inhibitors. Pulse-chase analysis showed that the cleaved form of TLR9 was still present in RAW-TLR9 cells treated with the broad spectrum inhibitors E64d, pepstatin A and leupeptin as well as with specific inhibitors of CTSK, CTSB and CTSS (Supplementary Fig. 4). With the exception of a CTSB inhibitor, these compounds did not affect TLR9 signalling (Supplementary Fig. 4 and data not shown). Finally, we observed no defect in TLR9 signalling in macrophages and dendritic cells from CTSS-, CTSS/ CTSL-, CTSK- and CTSB-deficient mice (Supplementary Fig. 5). Notably, these results disagree with recent work implicating CTSK and CTSB in TLR9 function ${ }^{13,14}$, although these studies relied heavily on protease inhibitors that may have off-target effects. Instead, our data suggest that none of these proteases is solely responsible for cleaving TLR9 and are consistent with an interpretation that multiple proteases are capable of processing the receptor.

The results described above strongly implicate proteolysis as a step required for TLR9 function, but they do not directly demonstrate that processed TLR9 is a functional receptor. To address this possibility, we tested whether the cleaved form of TLR9 can bind stimulatory $\mathrm{CpG}$ oligonucleotides. We found that TLR9 purified from phagosomes and incubated with biotinylated-CpG oligonucleotides could be efficiently precipitated using streptavidin beads (Fig. 4c). Thus, this processed form of TLR9, which is exclusively in the phagosome, is capable of recognizing ligand. We next compared CpG binding between the full-length and cleaved forms of TLR9. The truncated receptor was pulled down more efficiently than the fulllength receptor, suggesting that it may have a higher affinity for ligand (Fig. 4d). This interaction was specific, as binding was disrupted by addition of non-biotinylated $\mathrm{CpG}$ oligonucleotide (Fig. 4d).

Because both full-length and processed TLR9 can bind ligand, we sought to determine which form is responsible for signal transduction. The adaptor MyD88 associates with TLR9 after activation, so we examined which form of the receptor recruits MyD88 in response to CpG stimulation. Remarkably, immunoprecipitation of MyD88 in activated cells pulled down only the processed form of TLR9 (Fig. 4e). We were unable to detect any association between MyD88 and the full-length receptor, demonstrating that the processed form of TLR9 is the functional form of the receptor. We next hypothesized that requiring proteolysis may reduce the likelihood that functional receptors will leak to the cell surface and encounter self nucleic acids. To test this possibility, we used a recently reported strategy of fusing UCN93B1 to a yeast protein domain, Ist2, that directs transport of both UNC93B1 and TLR9 to the plasma membrane ${ }^{10}$. Whereas UNC93B1-Ist2 localized to the plasma membrane (Supplementary Fig. 6a), we did not observe significantly altered TLR9 processing or signalling in UNC93B1-Ist2 expressing cells (Fig. 4f and Supplementary Fig. 6b). These results suggest that a significant portion of TLR9 may avoid relocalization in UNC93B1-Ist2 cells and signal from internal compartments. Thus, we fused TLR9 directly to the Ist 2 secretion sequence and examined the signalling and localization of this fusion protein. Consistent with relocalization of the receptor to the cell surface, EndoH analysis indicated that the TLR9Ist2 fusion protein passed through the Golgi but remained unprocessed (Fig. 4g). Remarkably, TLR9-Ist2 no longer responded to CpG oligonucleotides (Fig. 4h). To ensure that this lack of signalling was not due to the Ist2 domain simply interfering with the TLR9 TIR domain, we generated a TLR9-Ist2 fusion protein in which the key amino acids necessary for plasma membrane targeting have been deleted (TLR9-Ist2 $(\Delta 18))^{15}$. This receptor was processed (Fig. 4g) and regained the ability to respond to CpG oligonucleotides (Fig. 4h), despite the presence of the non-functional Ist2 domain at its $\mathrm{C}$ terminus.

Our results reveal that compartmentalized proteolysis of TLR9 represents a key step in nucleic acid recognition. Although both full-length and processed forms of the receptor can bind ligand, only the cleaved form of TLR9 is competent for signal transduction. It remains unclear why processing is necessary for TLR9 activation. In a previous study we showed that a surface-localized chimaeric receptor TLR9N4C (consisting of the TLR9 ectodomain and TLR4 transmembrane and cytosolic domains) was hyper-responsive to self $\mathrm{DNA}^{4}$. Unlike TLR9-Ist2, this receptor signals from the cell surface even when fused to Ist2 but is not processed (Fig. 4h right panel and ref. 4). Swapping the transmembrane and cytosolic domains of TLR9 for those of TLR4 has apparently released this receptor from the processing requirement. Recent work suggests that TLR9 undergoes a membrane-proximal conformational change on ligand binding and activation $^{16}$. One possibility is that TLR9 cleavage is a prerequisite for this conformational change. This regulatory mechanism may not be required for cell surface receptors like TLR4, explaining the ability of full-length TLR9N4C to signal. Analyses comparing the structure and conformational changes of the full-length and truncated TLR9 receptors will be necessary to resolve these possibilities.

The proteolytic regulatory step described here is consistent with a model in which TLRs involved in nucleic acid sensing are translated as 'pro-receptors' in the ER and only function after being processed in the endolysosomal compartment (Fig. 4i). This scenario is 
a

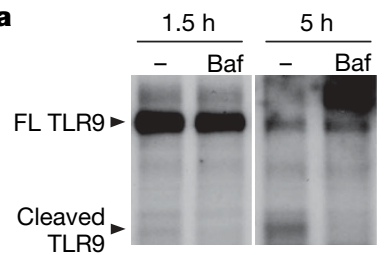

b
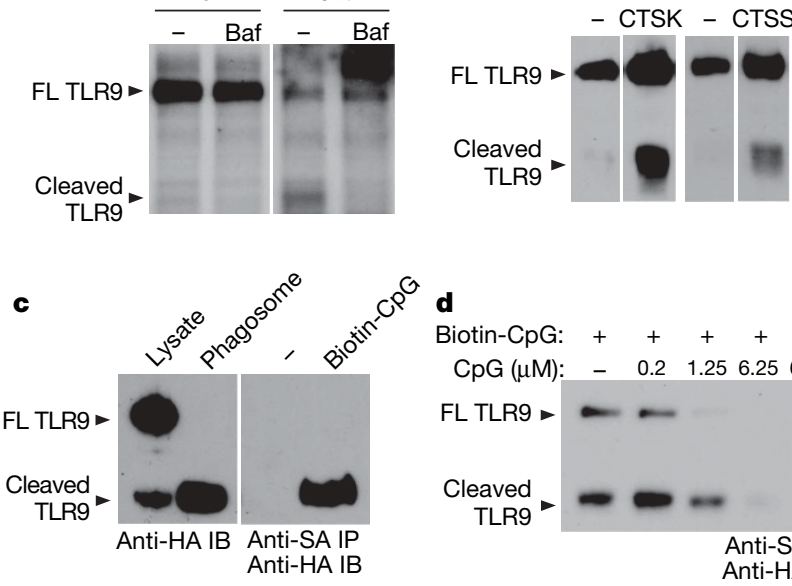

d
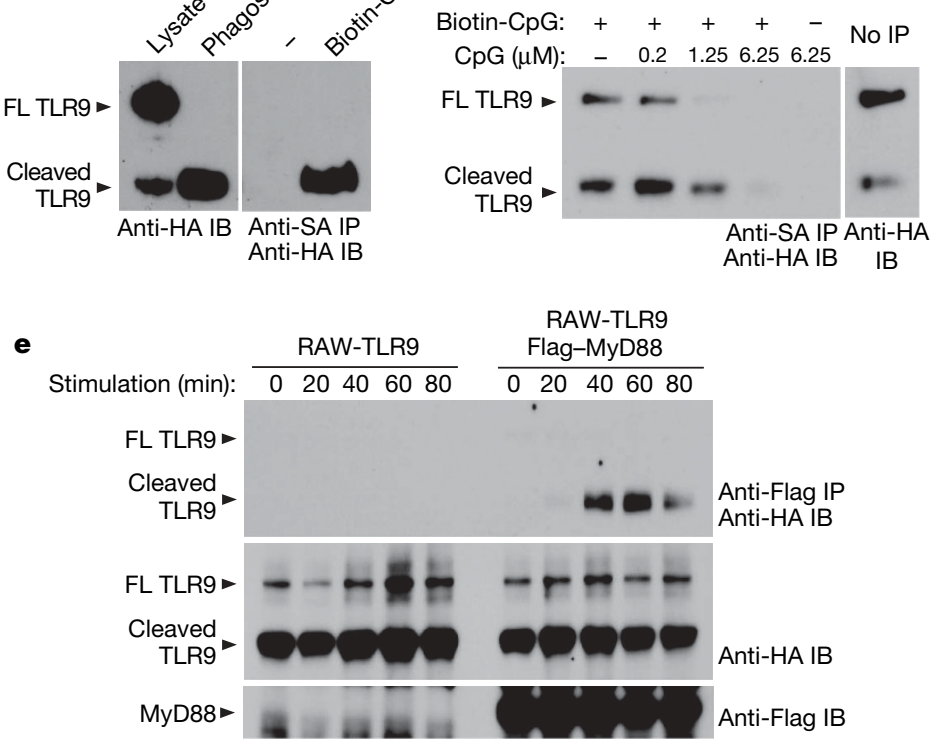

Figure 4 | Processing of TLR9 is necessary to generate a functional receptor. a, TLR9 cleavage requires the activity of acid-dependent proteases. Pulse-chase analysis was performed on RAW-TLR9 cells treated with $50 \mathrm{nM}$ bafilomycin A1 (Baf) or DMSO (-) vehicle control. b, In vitro proteolysis assay performed on TLR9-HA immunoprecipitated from 293T cells. TLR9-HA was incubated with recombinant CTSK, CTSS or no cathepsin (-) and detected by anti-HA immunoblot. $c$, The cleaved form of TLR9 can bind ligand. Left: whole-cell lysates or lysates of purified phagosomes were separated by SDS-PAGE and immunoblotted with anti-HA antibody. Right: lysates of purified phagosomes were incubated with $(+)$ or without $(-)$ $0.2 \mu \mathrm{M}$ biotin-CpG followed by streptavidin precipitation. TLR9-HA was detected by anti-HA immunoblot. Data are representative of two experiments. $\mathbf{d}$, Cell lysates were incubated with $(+)$ or without $(-)$ biotin$\mathrm{CpG}$ and precipitated with streptavidin (SA) as described in c. Where indicated, unconjugated $\mathrm{CpG}$ was also added, before precipitation. Unmanipulated lysate (No IP) is shown as a control. e, Cleaved TLR9

conceptually analogous to the production of proteases as proenzymes that must be processed before they gain enzymatic activity. In this way, compartmentalized processing of TLR9 may regulate where within the cell TLR9 activation can occur. We and others have argued that restricting activation of TLR9 and TLR7 to intracellular compartments may limit responses to self nucleic acids while preserving the ability to recognize foreign nucleic $\operatorname{acid}^{4,17,18}$. Thus, the requirement for processing of TLR9 and TLR7 may represent an evolutionary strategy to ensure proper self/non-self discrimination based on nucleic acid recognition.

\section{METHODS SUMMARY}

Detailed descriptions of reagents and experiments can be found in the full Methods. All stable cell lines were generated by retroviral gene transfer. For phagosome isolation, RAW cells were fed latex beads (Polysciences), the cells were disrupted by dounce homogenization, and latex-bead-containing phagosomes were purified on a $62-10 \%$ sucrose step gradient in a Beckman SW40Ti centrifuge. After purification, phagosomes were lysed by addition of Triton $\mathrm{X}-100$, and proteins in phagosomes were separated by SDS-polyacrylamide gel electrophoresis (PAGE) and visualized by immunoblot. Pulse-chase analyses
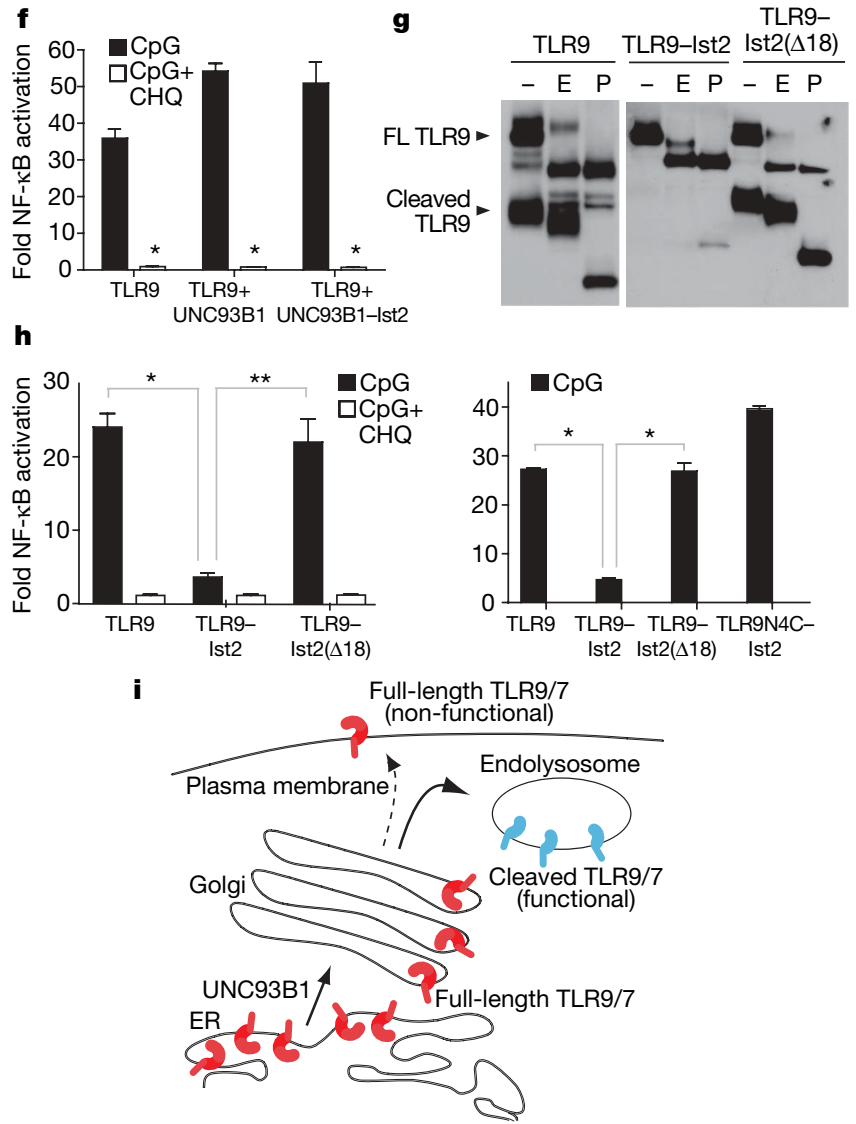

recruits MyD88. RAW cells expressing TLR9-HA and Flag-MyD88 were stimulated with $\mathrm{CpG}$ oligonucleotides followed by lysis at the indicated time points. After lysis, MyD88 was immunoprecipitated and TLR9 or MyD88 were detected by anti-HA or anti-Flag immunoblot, respectively. f, TLR9 does not signal from the cell surface in UNC93B1-Ist 2 cells. 293T cells were transiently transfected with an NF- $\kappa \mathrm{B}$ luciferase reporter together with the indicated expression plasmids. Luciferase production was assayed $12 \mathrm{~h}$ after stimulation with $3 \mu \mathrm{M} \mathrm{CpG}$, with or without pre-treatment with $50 \mu \mathrm{M}$ chloroquine (CHQ). g, TLR9, TLR9-Ist2 and TLR9-Ist2( $\Delta 18)$ expressed in RAW cells were analysed for sensitivity to EndoH or PNGaseF as described in Fig. 2a. h, 293T cells were transiently transfected with the indicated expression plasmids and $\mathrm{NF}-\kappa \mathrm{B}$ activation was measured as described in f. i, Schematic of compartmentalized proteolysis and regulation of TLR 9 and TLR7. For the indicated statistical comparisons, asterisk indicates $P<0.05$; double asterisk indicates $P<0.1$. Unless noted otherwise, all data presented are representative of at least three independent experiments.

were performed by starving cells of cysteine/methionine for $1 \mathrm{~h}$, pulsing with ${ }^{35} \mathrm{~S}$-cysteine/methionine for $45 \mathrm{~min}$, and chasing in molar excess of unlabelled cysteine/methionine for the indicated time periods. After lysis, radiolabelled proteins were immunoprecipitated and visualized by SDS-PAGE. Deglycosylation assays were performed on immunoprecipitated proteins using endoglycosidase H or PNGaseF (both from NEB) with supplied buffers according to manufacturer's instructions. Proteins were separated by SDS-PAGE and visualized by immunoblot. In vitro proteolysis with recombinant cathepsins (Biomol) was performed according to manufacturer guidelines. For MyD88/ TLR9 co-immunoprecipitations, cells were lysed in RIPA buffer before or after stimulation with $\mathrm{CpG}$, and protein complexes were precipitated with anti-Flag matrix. For CpG binding assays, TLR9-HA-containing lysates were incubated with biotin-CpG followed by precipitation with streptavidin matrix. In both cases, precipitated proteins were visualized by SDS-PAGE followed by immunoblot. NF- $\kappa \mathrm{B}$ luciferase assays were performed by transient transfection of HEK293T cells with an NF- $\mathrm{KB}$ luciferase reporter plasmid and the indicated expression plasmids using LTX transfection reagent (Invitrogen) according to manufacturer's instructions. C57/BL/6 mice were purchased from Jackson Laboratories. All knockout mice have been previously described ${ }^{19-22}$. Mice were housed within animal facilities at UC Berkeley or UCSF according to IACUC guidelines. 
Full Methods and any associated references are available in the online version of the paper at www.nature.com/nature.

Received 13 August; accepted 11 September 2008. Published online 28 September 2008.

1. Akira, S., Takeda, K. \& Kaisho, T. Toll-like receptors: critical proteins linking innate and acquired immunity. Nature Immunol. 2, 675-680 (2001).

2. Iwasaki, A. \& Medzhitov, R. Toll-like receptor control of the adaptive immune responses. Nature Immunol. 5, 987-995 (2004).

3. Marshak-Rothstein, A. Toll-like receptors in systemic autoimmune disease. Nature Rev. Immunol. 6, 823-835 (2006).

4. Barton, G. M., Kagan, J. C. \& Medzhitov, R. Intracellular localization of Toll-like receptor 9 prevents recognition of self DNA but facilitates access to viral DNA. Nature Immunol. 7, 49-56 (2006).

5. Latz, E. et al. TLR9 signals after translocating from the ER to CpG DNA in the lysosome. Nature Immunol. 5, 190-198 (2004)

6. Leifer, C. A. et al. TLR9 is localized in the endoplasmic reticulum prior to stimulation. J. Immunol. 173, 1179-1183 (2004).

7. Bell, J. K., Askins, J., Hall, P. R., Davies, D. R. \& Segal, D. M. The dsRNA binding site of human Toll-like receptor 3. Proc. Natl Acad. Sci. USA 103, 8792-8797 (2006).

8. Liu, L. et al. Structural basis of Toll-like receptor 3 signaling with double-stranded RNA. Science 320, 379-381 (2008).

9. Brinkmann, M. M. et al. The interaction between the ER membrane protein UNC93B and TLR3, 7, and 9 is crucial for TLR signaling. J. Cell Biol. 177, 265-275 (2007).

10. Kim, Y. M., Brinkmann, M. M., Paquet, M. E. \& Ploegh, H. L. UNC93B1 delivers nucleotide-sensing toll-like receptors to endolysosomes. Nature 452, 234-238 (2008)

11. Tabeta, K. et al. The Unc93b1 mutation 3d disrupts exogenous antigen presentation and signaling via Toll-like receptors 3, 7 and 9. Nature Immunol. 7, 156-164 (2006)

12. Hacker, H. et al. CpG-DNA-specific activation of antigen-presenting cells requires stress kinase activity and is preceded by non-specific endocytosis and endosomal maturation. EMBO J. 17, 6230-6240 (1998).

13. Asagiri, M. et al. Cathepsin K-dependent Toll-like receptor 9 signaling revealed in experimental arthritis. Science 319, 624-627 (2008).

14. Matsumoto, F. et al. Cathepsins are required for Toll-like receptor 9 responses. Biochem. Biophys. Res. Commun. 367, 693-699 (2008).
15. Juschke, C. Wachter, A., Schwappach, B. \& Seedorf, M. SEC18/NSF-independent, protein-sorting pathway from the yeast cortical ER to the plasma membrane. J. Cell Biol. 169, 613-622 (2005).

16. Latz, E. et al. Ligand-induced conformational changes allosterically activate Tolllike receptor 9. Nature Immunol. 8, 772-779 (2007).

17. Diebold, S. S., Kaisho, T., Hemmi, H., Akira, S. \& Reis e Sousa, C. Innate antiviral responses by means of TLR7-mediated recognition of single-stranded RNA. Science 303, 1529-1531 (2004)

18. Lund, J. M. et al. Recognition of single-stranded RNA viruses by Toll-like receptor 7. Proc. Natl Acad. Sci. USA 101, 5598-5603 (2004).

19. Deussing, J. et al. Cathepsins $B$ and $D$ are dispensable for major histocompatibility complex class II-mediated antigen presentation. Proc. Natl Acad. Sci. USA 95, 4516-4521 (1998).

20. Mallen-St \& Clair, J. et al. Cathepsins $L$ and $S$ are not required for activation of dipeptidyl peptidase I (cathepsin C) in mice. Biol. Chem. 387, 1143 (2006).

21. Saftig, P. et al. Impaired osteoclastic bone resorption leads to osteopetrosis in cathepsin-K-deficient mice. Proc. Natl Acad. Sci. USA 95, 13453-13458 (1998).

22. Shi, G.-P. et al. Cathepsin S required for normal MHC class II peptide loading and germinal center development. Immunity 10, 197-206 (1999).

Supplementary Information is linked to the online version of the paper at www.nature.com/nature.

Acknowledgements We thank members of the Barton and Vance laboratories for discussions, S. Akira for providing TLR knockout mice, and R. Vance and R. Medzhitov for advice. We acknowledge the CHPS Imaging Core and P. Herzmark for assistance with microscopy. This work was supported by a Novel Research Grant from The Lupus Research Institute (G.M.B.), the Hellman Faculty Fund (G.M.B.), and NIH grants HL67204 (H.A.C.), CA009179 (S.E.E.) and AI072429 (G.M.B.).

Author Contributions S.E.E. and G.M.B. planned experiments and wrote the manuscript. S.E.E., B.L.L., L.L. and K.E.W. performed the experiments. G.-P.S. and H.A.C. contributed reagents. All authors read and commented on the manuscript.

Author Information Reprints and permissions information is available at www.nature.com/reprints. Correspondence and requests for materials should be addressed to G.M.B. (barton@berkeley.edu) 


\section{METHODS}

Reagents. All chemicals and reagents, unless noted otherwise, were purchased from Fisher Scientific. Anti-HA (clone 3F10) and anti-Flag (M2) affinity matrices were purchased from Roche and Sigma, respectively. Latex beads were purchased from Polysciences. CpG oligonucleotides (TCCATGACGTTCCTGACGTT) with phosphorothioate linkages and 5'-biotinylated CPG oligonucleotides (of the same sequence) were purchased from Invitrogen and IDT. LPS, R848, BLP and biotinylated LPS were purchased from Invivogen. All antibodies for flow cytometry and ELISAs were purchased from eBioscience. The following antibodies were used for immunoblotting: anti-HA (Roche), anti-Flag M2 (Sigma), anti-calnexin (Stressgen), anti-HSP90B (Thermo Scientific), antiLAMP1 (Santa Cruz), anti-tubulin (EMD), anti-nuclear pore complex (Covance), goat anti-rat-HRP and sheep anti-mouse HRP (GE Amersham).

Mice. C57BL/6 and 129Sv mice were purchased from Jackson Laboratories. Tlr $2^{-1-} \mathrm{Tlr} 4^{-1-} \mathrm{Tlr} 9^{-1-}$ mice were generated by intercrossing individual knockout mice, provided by S. Akira. Ctsk ${ }^{-1-}$ (ref. 21), $\mathrm{Ctss}^{-1-}$ (ref. 22) and $\mathrm{Ctss}^{-1-} \mathrm{Ctsl}^{-1-}$ (ref. 20) mice (all backcrossed to C57BL/6) and $\mathrm{CtsB}^{-1-}$ (ref. 19) mice (on the 129Sv background) have been previously described. All mice were housed within the animal facilities at the University of California at Berkeley or University of California at San Francisco according to IACUC guidelines.

Cell lines, plasmids and tissue culture. RAW264 cells were purchased from ATCC and cultured in RPMI supplemented with $10 \%$ FCS, L-glutamine, penicillin/streptomycin, sodium pyruvate and HEPES (all from Invitrogen). Unless otherwise noted, stable lines were generated by transducing cells with MSCV2.2 retroviruses encoding the target cDNA. For RAW-TLR9, RAW-TLR7 and RAWTLR4 cells, cDNA encoding the HA epitope was inserted at the $3^{\prime}$ end of each TLR. Flag-TLR9 and Flag-MyD88 have been previously described ${ }^{23}$. UNC93BIst 2 was constructed as previously described ${ }^{10}$. TLR9-Ist2 and TLR9N4C-Ist2 were made by fusing the 69-residue Ist2 tag to the $3^{\prime}$ end of TLR9-HA or TLR9N4C-HA, respectively.

Bone-marrow-derived conventional dendritic cells, macrophages and plasmacytoid dendritic cells were differentiated, as previously described ${ }^{4}$, in RPMI supplemented with GM-CSF containing supernatant, M-CSF containing supernatant, or $25 \mathrm{ng} \mathrm{ml}^{-1}$ recombinant Flt3L (R\&D), respectively. To generate conventional dendritic cells and plasmacytoid dendritic cells expressing TLR9-HA, haematopoietic stem cells (HSCs) from $\mathrm{Tlr}^{-1-} \mathrm{Tlr}^{-1-} \mathrm{Tlr}^{-1-}$ mice were transduced with retroviruses encoding TLR9-HA as previously described ${ }^{4}$. Briefly, mice were injected with 5-flourouracil (Sigma) to enrich for HSCs. HSCs were cultured in IL-6, IL-3 and SCF (all from R\&D) over 4 days and transduced with retroviruses. After 5 days, HSCs were differentiated into conventional dendritic cells or plasmacytoid dendritic cells as described above.

Splenic dendritic cells were isolated from C57BL/6 or $\mathrm{Ctsk}^{-1-}$ mice by magnetic cell sorting using anti-CD11c conjugated magnetic beads (Miltenyi Biotech) and an AutoMACS cell sorter (Miltenyi Biotech). Enrichment, as measured by FACS, was greater than $70 \%$.

Immunoprecipitation and western blot analyses. Cells were lysed in RIPA buffer ( $50 \mathrm{mM}$ Tris $\mathrm{pH} 8,150 \mathrm{mM} \mathrm{NaCl}, 1 \% \mathrm{NP}-40,0.5 \%$ sodium deoxycholate, $0.1 \%$ SDS) supplemented with Complete protease inhibitor cocktail (Roche). After incubation on ice, lysates were cleared of insoluble material by centrifugation. For immunoprecipitations, lysates were incubated with anti-HA matrix or anti-Flag matrix and precipitated proteins were separated by SDS-PAGE and transferred to Immobilon PVDF membrane (Millipore). Membranes were probed with the indicated antibodies and developed by ECL chemiluminescence (Pierce). Relative band intensities were quantified by counting pixels per band using ImageJ software.

Steptavidin pull downs were performed on cells or purified phagosomes lysed in TNT buffer (20 mM Tris pH 8.0, $200 \mathrm{mM} \mathrm{NaCl}, 1 \%$ Triton X-100) and cleared of insoluble debris. Lysates were incubated for $1 \mathrm{~h}$ with $0.2 \mu \mathrm{M}$ biotin-CpG and/ or unlabelled $\mathrm{CpG}$ on ice, added to streptavidin-agarose (Invitrogen, preblocked with $1 \%$ BSA-PBS), rotated $1 \mathrm{~h}$ at $4{ }^{\circ} \mathrm{C}$, and washed four times in lysis buffer. Precipitates were boiled in SDS buffer, separated by SDS-PAGE, and probed by anti-HA immunoblot.

Microscopy. Co-localization studies were performed on a Zeiss 510 Axioplane META microscope. Coverslips were coated with poly-D-lysine (Sigma), washed extensively with water and allowed to dry $(1 \mathrm{~h})$. Cells were allowed to settle overnight. The next day, coverslips were washed with PBS, fixed with $4 \%$
PFA/PBS, and permeabilized with $0.5 \%$ Triton X-100/PBS. After washing with $0.1 \%$ Triton X-100/PBS, cells were blocked (5\% goat sera, $2 \%$ BSA, $0.1 \%$ sodium azide, $0.1 \%$ Triton X-100/PBS). Slides were stained in blocking buffer with antiFlag, anti-HA, anti-calnexin or anti-LAMP1 (see antibodies used for western blot). Secondary antibodies were donkey anti-rabbit Cy5, donkey anti-rabbit $\mathrm{Cy} 3$, goat anti-rat $\mathrm{Cy} 3$, goat anti-mouse Cy3 (Jackson) or donkey anti-rat Alexa680 (Invitrogen).

Visualization of UNC93B1-GFP and UNC93B1-GFP-Ist2 was performed on a Nikon E800 fluorescent microscope. Cells were plated and fixed as described above.

Luciferase assays. Transient transfections were performed in OptiMem (Invitrogen) with LTX transfection reagent (Invitrogen) according to manufacturer's guidelines. 293T cells were transiently co-transfected with the indicated expression plasmid(s) and a plasmid encoding an NF- $\kappa \mathrm{B}$ luciferase reporter (with five tandem NF- $\kappa \mathrm{B}$ binding sites, provided by R. Vance). Cells were stimulated with $3 \mu \mathrm{M} \mathrm{CpG}$ oligonucleotide after $24 \mathrm{~h}$ and lysed by passive lysis after an additional $12 \mathrm{~h}$. Luciferase activity was measured on a LMaxII-384 luminometer (Molecular Devices).

Pulse-chase. Cells were starved for $1 \mathrm{~h}$ in cysteine/methionine-free media, then pulsed with $0.1 \mathrm{mCi}{ }^{35} \mathrm{~S}$-cysteine/methionine (Perkin-Elmer). After a 45 -min pulse, cells were washed and cultured in $5 \mathrm{ml}$ chase media with 10,000-fold molar excess of L-cysteine, L-methionine or harvested as the zero time point. Time points were harvested as follows: cells were washed twice in $2 \mathrm{ml}$ PBS and lysed in $1 \mathrm{ml}$ RIPA plus protease inhibitor cocktail. For protease inhibitor experiments, inhibitors were added in chase media and supplemented every $2 \mathrm{~h}$ at the following concentrations: $50 \mu \mathrm{M}$ E64d (EMD), $100 \mu \mathrm{M}$ pepstatin A (Sigma), $100 \mu \mathrm{M}$ leupeptin (Sigma), $10 \mathrm{nM}$ cathepsin $\mathrm{K}$ inhibitor III (CbzLeu-NH- $\mathrm{CH}_{2}-\mathrm{CO}-\mathrm{CH}_{2}-\mathrm{NH}-\mathrm{Leu}-\mathrm{Cbz}$, Sigma), $30 \mu \mathrm{M}$ CtsB inhibitor (Me047, EMD), $2 \mu \mathrm{M}$ CtsS inhibitor (-Phe-Leu-COCHO$\cdot \mathrm{H}_{2} \mathrm{O}, \mathrm{EMD}$ ). Baflomycin A1 (Sigma) was used at $50 \mathrm{nM}$ and brefeldin A (Sigma) was used at $10 \mu \mathrm{g} \mathrm{ml}^{-1}$.

Phagosome isolation. $4 \times 10^{7} \mathrm{RAW}$ cells were incubated with $\sim 10^{8} 2 \mu \mathrm{M}$ latex beads (Polysciences) for various time points. After rigorous washing in PBS, RAW cells were scraped into $3 \mathrm{ml}$ sucrose homogenization buffer (SHB: $250 \mu \mathrm{M}$ sucrose, $3 \mathrm{mM}$ imidazole $\mathrm{pH}$ 7.4) and pelleted by centrifugation. Cells were resuspended in $1 \mathrm{ml} \mathrm{SHB}$ plus protease inhibitor cocktail (Roche) and EDTA and disrupted by 25 strokes in a steel dounce homogenizer. The disrupted cells were gently rocked for $10 \mathrm{~min}$ on ice and centrifuged at $8,000 \mathrm{~g}$ to remove intact cells, nuclei and gross debris. The remaining supernatant was further processed to separate intact phagosomes. A total of $40 \mu \mathrm{l}$ was set aside as the 'lysate' sample and lysed by addition of $1 \%$ Triton X-100. The remaining supernatant was mixed with an equal volume of $60 \%$ sucrose, and applied to a sucrose step gradient as follows: $1 \mathrm{ml} 60 \%$ sucrose, $2 \mathrm{ml}$ homogenate, $2 \mathrm{ml} \mathrm{32 \%} \mathrm{sucrose,}$ $2 \mathrm{ml} 20 \%$ sucrose, $2 \mathrm{ml} 10 \%$ sucrose. The gradient was spun at $100,000 \mathrm{~g}$ for $1 \mathrm{~h}$ in a SW40 rotor. Following centrifugation, phagosomes were harvested from the $20-10 \%$ sucrose interface. Phagosomes were diluted in $10 \mathrm{ml}$ PBS and spun at $40,000 \mathrm{~g}$ for $15 \mathrm{~min}$ in an SW40 rotor. The phagosome pellet was resuspended in $40 \mu \mathrm{l} \mathrm{SHB}$, protease inhibitor cocktail, EDTA and 1\% Triton X-100. Protein concentrations were normalized by BCA assay, and lysates were analysed by western blot.

In vitro proteolysis assay. Recombinant cathepsin $\mathrm{S}$ and recombinant procathepsin K were purchased from BioMol. 293T cells stably expressing TLR9HA were lysed in RIPA buffer and TLR9 was immunoprecipitated with anti-HA agarose. Immunoprecipitates were washed four times in cathepsin reaction buffer ( $50 \mathrm{mM}$ potassium phosphate $\mathrm{pH} 6.5,50 \mathrm{mM} \mathrm{NaCl}, 2 \mathrm{mM}$ EDTA, $0.01 \%$ Triton X-100, $0.5 \mathrm{mM}$ dithiothreitol) followed by incubation with or without cathepsins. Pro-cathepsin K was activated according to manufacturer's instructions (that is, incubation in $32.5 \mathrm{mM} \mathrm{NaOAc} \mathrm{pH} 3.5$ for $25 \mathrm{~min}$ at $24^{\circ} \mathrm{C}$ ). Activated cathepsin $\mathrm{K}$ was immediately incubated with immunoprecipitated TLR9-HA in cathepsin reaction buffer for $45 \mathrm{~min}$ at $24^{\circ} \mathrm{C}$. Cathepsin S (0.0024 units) was added directly to immunoprecipitated TLR9-HA in cathepsin reaction buffer and incubated for $45 \mathrm{~min}$ at $40^{\circ} \mathrm{C}$. Enzymes were inactivated by the addition of SDS loading buffer and boiling. Reactions were resolved by SDS-PAGE and anti-HA immunoblot (as described above).

23. Horng, T., Barton, G. M. \& Medzhitov, R. TIRAP: an adapter molecule in the Toll signaling pathway. Nature Immunol. 2, 835-841 (2001). 